\title{
C2 rs547154 polymorphism and polypoidal choroidal vasculopathy susceptibility: a meta-analysis
}

SUBJECT AREAS:

GENETIC MARKERS

GENETICS RESEARCH

GENETIC TESTING

Received

4 December 2014

Accepted

2 February 2015

Published

3 March 2015

Correspondence and requests for materials should be addressed to

C.Z. (dr_zhaochen@

163.com)

\author{
Xue Chen' ', Xiaoli Kang ${ }^{2}$, Kanxing Zhao ${ }^{3}$ \& Chen Zhao'
}

'Department of Ophthalmology, The First Affiliated Hospital of Nanjing Medical University, State Key Laboratory of Reproductive Medicine, Nanjing, China, ${ }^{2}$ Department of Ophthalmology, Xinhua Hospital, Shanghai Jiao Tong University School of Medicine, Shanghai, China, ${ }^{3}$ Tianjin Medical University, Tianjin Eye Hospital, Tianjin Key Laboratory of Ophthalmology and Visual Science, Tianjin, China.

Previous studies have indicated the association between $C 2$ rs547154 polymorphism and polypoidal choroidal vasculopathy (PCV) risk, while the results are controversial and inconsistent. Herein, we perform a meta-analysis to gain a precise estimation of the association using 5 eligible studies involving 4076 subjects, of which 1220 were PCV cases, 1073 were age-related macular degeneration (AMD) cases and 1783 were controls. Allelic frequencies of $C 2$ rs547154 polymorphism between PCV and AMD were also compared. Both crude and adjusted odds ratios (OR) with their $95 \%$ confidence interval (CI) were included to assess the strength of the association. The pooled $\mathrm{OR}$ in random-effect model for allele $\mathrm{T}$ versus $\mathrm{G}$ was 0.64 (95\% CI, $0.52-0.80 ; p<0.0001)$, for genotype TG versus GG was 0.65 (95\% CI, $0.52-0.83 ; p, 0.0004)$, and for genotype TT + TG versus GG was $0.64(95 \% \mathrm{CI}, 0.51-0.80 ; p, 0.0002)$. No difference in allelic frequency was observed between PCV and AMD (OR, 0.86; 95\% CI, 0.64-1.16; $p, 0.32$ ). Sensitivity analysis proved the robustness of our data. No significant ethnic divergence was suggested by subgroup analysis, and no publication bias was detected via Egger's test. In conclusion, our data indicate that $C 2$ rs547154 polymorphism plays a protective role in the development of PCV.

$\mathrm{P}$ olypoidal choroidal vasculopathy (PCV) is a hemorrhagic and exudative maculopathy characterized by hyalinization and peripapillary, macular or peripheral sub-retinal pigment epithelium (RPE) polypoidal dilatations of branching choroidal vascular networks in indocyanine green angiography (ICGA) presentations $s^{1-3}$, which may subsequently lead to detachments of RPE, or even neurosensory retina ${ }^{2,4-7}$. Due to the many shared clinical hallmarks with age-related macular degeneration (AMD), including recurrent subretinal hemorrhage, exudation, serous and hemorrhagic RPE detachment, and vitreous hemorrhage, PCV remains unrecognized for a long period until its first identification by Yannuzzi et al in $1990^{2}$. Recent studies indicate more unique manifestations in PCV that distinguished PCV from AMD, suggesting that PCV is a clinical entity separated from $\mathrm{AMD}^{1,8,9}$

The pathogenesis of PCV remains elusive, while it is widely accepted that both genetic and environmental factors play important roles in the disease course of PCV. Considering the clinical similarities between AMD and PCV, researchers aim to find out whether AMD and PCV share common pathogenic pathways. Previous metaanalyses have reported that age-related maculopathy susceptibility 2 (ARMS2; MIM: 611313) rs10490924 and complement factor H (CFH; MIM: 134370) rs800292 polymorphisms, variants implicated in AMD etiology, might also increase PCV risk ${ }^{10,11}$. The complement component 2 (C2; MIM: 613927) rs547154 variant is a G to T substitution located on chromosome $6 \mathrm{p} 21$, which shows protective effect against $\mathrm{AMD}^{12,13}$. This variant has been widely investigated for its association with $\mathrm{PCV}^{14-18}$, while the results are controversial and the sample size in each study is limited. Therefore, we performed this meta-analysis to assess the relationship between the $C 2$ variant and PCV susceptibility.

\section{Results}

Literature. The initial literature search yielded 97 articles relevant to the search terms. Five articles, published from 2008 to 2014, embodying 4076 subjects (1220 PCV cases, 1073 AMD cases and 1783 controls) were finally included for the meta-analysis ${ }^{14-18}$. Flow chart of literature screening and review was shown in Figure 1. All five were case-control studies in English with their characteristics listed in Table 1. The average ages ranged from 


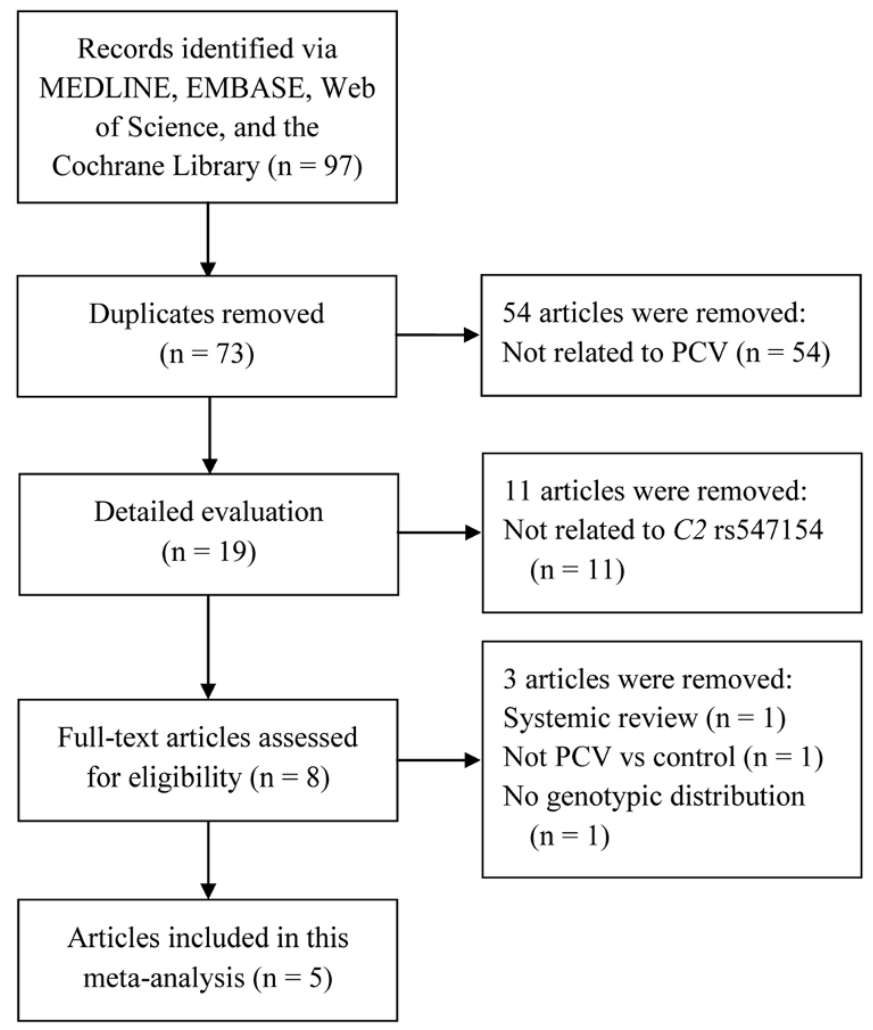

Figure $1 \mid$ Flow chart depicting the screening process for inclusion in the meta-analysis.

63.80 to 73.00 years in the PCV case group, from 73.60 to 75.59 years in the AMD case group, and from 48.22 to 72.90 years in the control group, while the gender ratios (male/female) in the three groups varied from $1.39(32 / 23)$ to $3.39(105 / 31)$ for PCV cases, $2.64(330 /$ $125)$ to $2.97(187 / 63)$ for AMD cases, and $0.67(110 / 164)$ to $1.51(110 /$ 73) for controls (Table 1). Of the 5 studies, 3 were in Japan ${ }^{15,17,18}, 1$ was in Singapore ${ }^{14}$, and another one was in $\mathrm{USA}^{16}$. Three studies used TaqMan for genotyping ${ }^{15,17,18}$ and one used Sanger sequencing ${ }^{14}$, whereas both polymerase chain reaction-restriction fragment length polymorphism (PCR-RFLP) and TaqMan were applied in the study performed by Lima et $\mathrm{al}^{16}$.

Meta-analysis. Allelic and genotypic distributions for the $C 2$ rs547154 variant from each individual study are presented in Table 2. The genotypic distribution in the control group was consistent with Hardy-Weinberg equilibrium (HWE) in each study. The adjusted odds ratios (OR) with their 95\% confidence interval (CI) and the corresponding adjusted variables were provided by two studies and were listed in Table $2^{17,18}$. Quality assessments of all included studies with the Newcastle-Ottawa Scale were included in Table S1.

In the random-effect model, the pooled $\mathrm{OR}$ for the risk allele $\mathrm{T}$ versus the wild type $\mathrm{G}$ was 0.64 (95\% CI, 0.52-0.80; $p<0.0001$ ) (Figure 2A), pooled OR for genotype TT versus GG was 0.43 (95\% CI, $0.15-1.26 ; p, 0.123)$, pooled OR for genotype TG versus GG was 0.65 (95\% CI, $0.52-0.83 ; p, 0.0004)$, pooled OR for genotype TT + TG versus GG was 0.64 (95\% CI, $0.51-0.80 ; p, 0.0002)$, and pooled OR for genotype TT versus TG + GG was 0.46 (95\% CI, 0.16-1.34; $p$, 0.154 ) (Table 3 ). No significant heterogeneities exist within all five comparison groups, and results from the fixed-effect model kept consistent with the random-effect model. To minimize the bias, we used adjusted data for better estimation, and the adjusted OR for $\mathrm{T}$ versus $\mathrm{G}$ was 0.56 (95\% CI, 0.42-0.74; $p<0.0001$ ) (Figure 2B). No difference in allelic frequency was found between the PCV and AMD

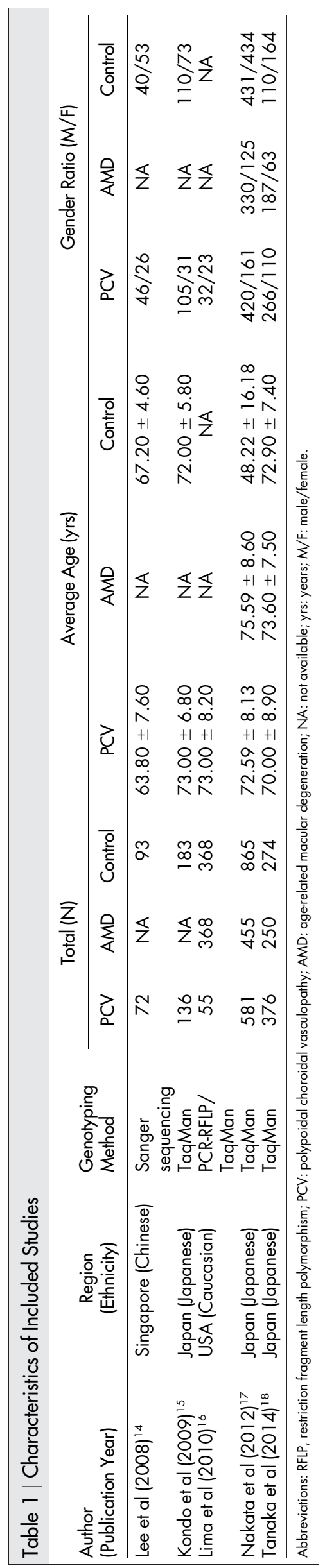


Table 2 | Genotype Distribution and Allele Frequency of Included Studies

\begin{tabular}{|c|c|c|c|c|c|c|c|c|c|c|c|c|}
\hline \multirow[b]{2}{*}{ Author } & \multicolumn{4}{|c|}{ PCV } & \multicolumn{4}{|c|}{ Control } & \multirow[b]{2}{*}{ HWE $p$ Value } & \multicolumn{2}{|c|}{ Adjusted Results (T vs G) } & \multirow[b]{2}{*}{ Adjusted Factors } \\
\hline & (N) & $\pi / T G / G G$ & $T / G$ & $\mathrm{~T}(\%)$ & $(\mathrm{N})$ & TT/TG/GG & $T / G$ & $\mathrm{~T}(\%)$ & & OR $[95 \% \mathrm{Cl}]$ & $p$ Value & \\
\hline Lee et $a^{14}$ & 72 & $0 / 5 / 67$ & $5 / 139$ & 3.47 & 93 & $0 / 9 / 84$ & $9 / 177$ & 4.84 & 0.624 & NA & NA & NA \\
\hline Kondo et al ${ }^{15}$ & 136 & $1 / 19 / 116$ & $21 / 251$ & 7.72 & 183 & $2 / 32 / 149$ & $36 / 330$ & 9.84 & 0.848 & NA & NA & NA \\
\hline Lima et al ${ }^{16}$ & 55 & $0 / 4 / 51$ & $4 / 106$ & 3.63 & 368 & $5 / 77 / 286$ & $87 / 649$ & 11.82 & 0.943 & NA & NA & NA \\
\hline
\end{tabular}

groups (OR, 0.86; 95\% CI, 0.64-1.16; $p, 0.32$ ) (Figure 2C). Subgroup analysis based on ethnicity revealed that results from both the Asian and the Caucasian groups kept consistent with overall data in all five tested models, suggesting no existed ethnic divergence (Table 3). Sensitivity analysis was applied to estimate the influence of each study on the pooled OR. No individual study was found to affect the result in all comparison groups, which further proved the robustness of our data. Further, no

\begin{tabular}{|c|c|c|c|c|c|}
\hline & Case & & Contr & & \\
\hline Study or Subgroup & Events & Total & Events & Total & Weig \\
\hline Lee 2008 & 5 & 144 & 9 & 186 & $3 . \subseteq$ \\
\hline Kondo 2009 & 21 & 272 & 36 & 366 & 15.4 \\
\hline Lima 2010 & 4 & 110 & 87 & 736 & 4.6 \\
\hline Nakata 2012 & 61 & 1162 & 131 & 1730 & 49.5 \\
\hline Tanaka 2014 & 42 & 752 & 49 & 548 & 26.6 \\
\hline Total $(95 \% \mathrm{Cl})$ & & 2440 & & 3566 & 100. \\
\hline Total events & 133 & & 312 & & \\
\hline
\end{tabular}

Heterogeneity: $\mathrm{Tau}^{2}=0.00 ; \mathrm{Chi}^{2}=3.13, \mathrm{df}=4(\mathrm{P}=0.54) ; \mathrm{I}^{2}=0 \%$

Test for overall effect: $Z=3.92(P<0.0001)$
Odds Ratio

M-H, Random, 95\% Cl Year

$0.71[0.23,2.16] 2008$

$0.77[0.44,1.35] 2009$

$0.28[0.10,0.78] 2010$

$0.68[0.49,0.93] 2012$

$0.60[0.39,0.92] 2014$

$0.64[0.52,0.80]$

\section{B}

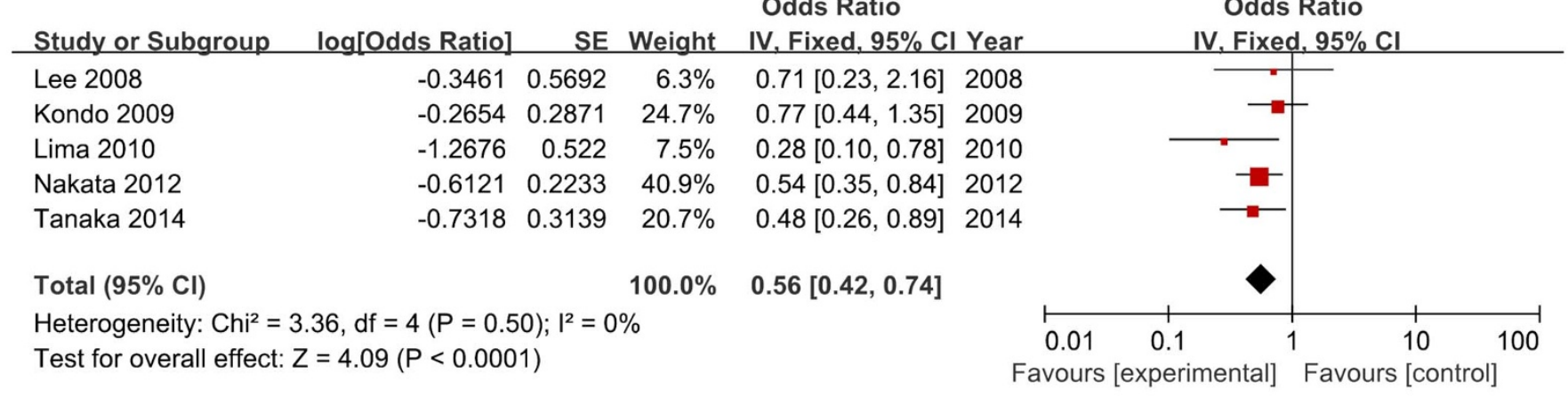

\section{C}

\begin{tabular}{|c|c|c|c|c|}
\hline Study or Subgroup & log[Odds Ratio] & SE & Weight & IV. Fixed, $95 \% \mathrm{CI}$ \\
\hline Lima 2010 & -0.2957 & 0.5171 & $8.5 \%$ & $0.74[0.27,2.05]$ \\
\hline Nakata 2012 & -0.1783 & 0.2048 & $54.1 \%$ & $0.84[0.56,1.25]$ \\
\hline Tanaka 2014 & -0.0761 & 0.2463 & $37.4 \%$ & $0.93[0.57,1.50]$ \\
\hline Total $(95 \% \mathrm{Cl})$ & & & $100.0 \%$ & $0.86[0.64,1.16]$ \\
\hline
\end{tabular}

Figure $2 \mid$ Association between C2 rs547154 polymorphism and polypoidal choroidal vasculopathy (PCV)/age-related macular degeneration (AMD) risk. Forest plots of $C 2$ rs547154 polymorphism (T vs G) and PCV risk based on crude (A) and adjusted data (B). Allelic frequencies of C2 rs547154 polymorphism (T vs G) between PCV and AMD (C). 


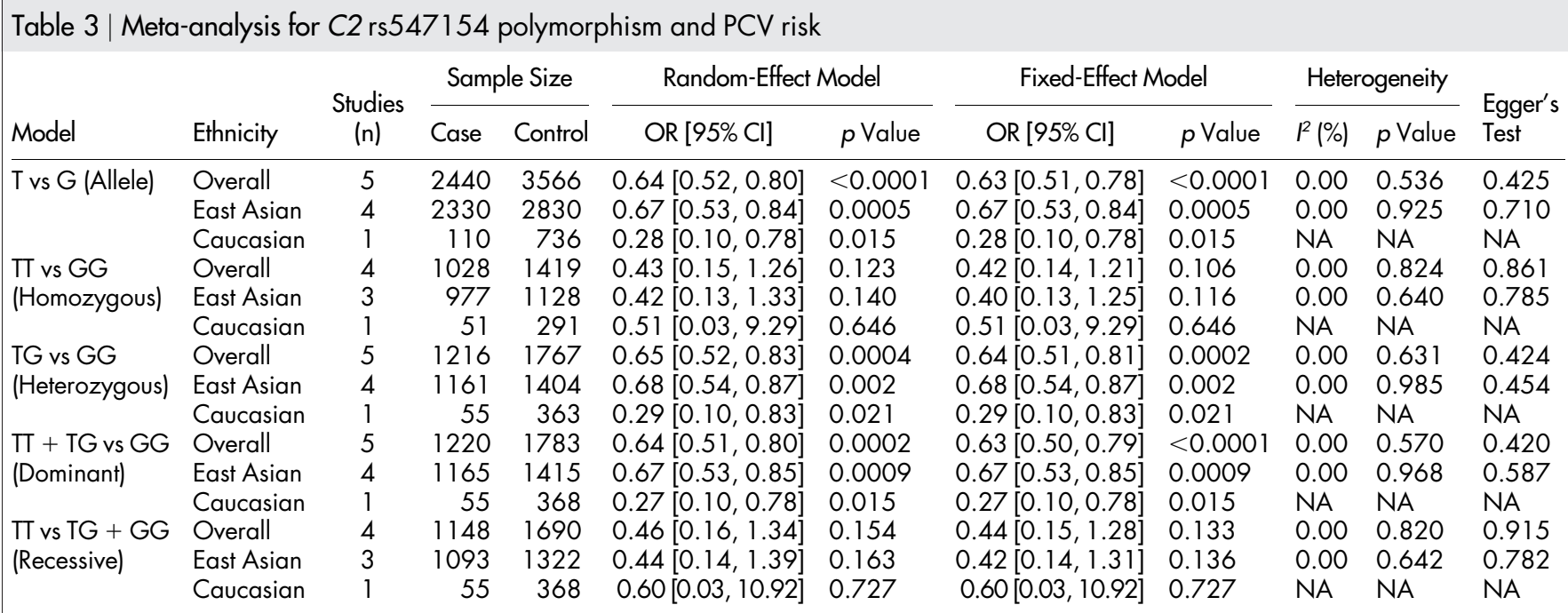

Abbreviations: PCV: polypoidal choroidal vasculopathy; OR: odds ratio; $\mathrm{Cl}$ : confidence interval.

publication bias was detected via Begg's funnel plot and Egger's test.

\section{Discussion}

Inconsistencies exist in the association between $C 2$ rs547154 polymorphism and PCV risk, suggesting the necessity to perform an exhaustive review and quantitative analysis on all evidence to determine the effect. In the present study, to assess the association between C2 rs547154 polymorphism and PCV risk, we reviewed a total of 97 published reports and completed an analysis on 4076 subjects from 5 original studies. Our result suggests that $C 2$ rs547154 has protective effect on PCV in all populations.

The $C 2$ locus and the $C F B$ locus are located closely together on chromosome $6 \mathrm{p}^{19}$. Primary structure of the C2 gene showed $39 \%$ sequence identity with its functionally analogous complement factor $\mathrm{B}$ (CFB; MIM: 138470) ${ }^{20}$. Component C2, protein encoded by the $C 2$ gene, shows a wide expression in the neural retina, RPE, and choroid. $\mathrm{C} 2$ is part of the classical pathway of the complement system. Upon the binding with component $\mathrm{C} 4 \mathrm{~b}$, component $\mathrm{C} 2$ is cleaved by activated factor component $\mathrm{C} 1$ into two fragments, $\mathrm{C} 2 \mathrm{~b}$ and $\mathrm{C} 2 \mathrm{a}^{21}$. The former fragment, $\mathrm{C} 2 \mathrm{~b}$, then combines with the complement factor $\mathrm{C} 4 \mathrm{~b}$ to generate the $\mathrm{C} 3 / \mathrm{C} 5$ convertase. Mutations in $\mathrm{C} 2$ have been implicated in causing complement component 2 deficiency (C2D; MIM: 217000$)^{22,23}$, whereas the functional role of $C 2$ in the etiology of PCV and AMD has not been fully elucidated. Thus, more functional investigations are warranted to give a better insight into the relationship between the $C 2$ variant and PCV.

To enhance the reliability of our results, we adopted the quality assessment tool recommended by the Newcastle-Ottawa Scale (NOS) for case-control studies. No language limitation was applied. Only studies published in peer-reviewed journals were included. In addition, we also used adjusted data for better estimation of the association. The association between C2 rs547154 polymorphism and PCV susceptibility has been discussed in a previous report, while they have only included 3 studies and tested the allele model ( $T$ versus $G)^{24}$. Several limitations of the present study should be acknowledged. The number of included studies was quite limited, and only one report was on Caucasians. Meanwhile, one study was not included due to the lack of allelic and genotypic data ${ }^{25}$. Therefore, more investigations are warranted to confirm the conclusion, especially in Caucasians and other populations. Despite our effort to narrow down the influence of other risk factors using adjusted data, the number of studies providing adjusted data, especially multivari- ate-adjusted data, was quite limited. We only conducted subgroup analysis on ethnicity, while the effects of age and gender were not evaluated due to the incomplete data in the control group.

In conclusion, our study suggests that the $C 2$ rs547154 variant shows a protective effect on the development of PCV. No significant difference was detected between the allelic frequencies in the PCV and the AMD groups. More epidemiological and biological studies are needed to ascertain the association, and to help to understand the molecular basis behind this association.

\section{Methods}

Literature Searching and Study Eligibility. This meta-analysis was performed according to the PRISMA guidelines ${ }^{26}$. We searched MEDLINE, EMBASE, Web of Science, and the Cochrane Library for all relevant articles using the following three main search terms as indicated previously ${ }^{11}$ : (1) ((("Choroid Diseases"'[Mesh] AND "Vascular Diseases"[Mesh]) AND polypoidal) OR (polypoidal choroidal vasculopathy OR PCV)) AND ((complement component 2 OR C2 OR) AND (IVS10 OR rs547154))); (2) ("polypoidal choroidal vasculopathy"/exp OR PCV AND ("complement component 2" OR C2) AND (IVS10 OR rs547154)); and (3) ((polypoidal choroidal vasculopathy) OR PCV) AND ((complement component 2 OR C2) AND (IVS10 OR rs547154)). The most recent search was performed on Nov 28,2014 . No language filters were applied. Retrieved records and eligibility status were managed using EndNote X5 software (http://endnote.com/).

Included studies were: (1) studies evaluating the association between C2 rs547154 polymorphism and PCV risk; (2) genome-wide association studies, or studies of casecontrol, cohort or population-based epidemiologic design; (3) studies using predefined criteria and procedures for PCV and AMD diagnoses; (4) OR have been reported, or present allelic and genotypic distributions of the $C 2$ rs547154 variant in PCV cases and control subjects that enable calculations of the outcomes. Alleles included T and G, and genotypes covered TT, TG and GG. We excluded case reports, reviews, conference proceedings, editorials, reports with incomplete data, and only included those with the latest follow-up information for serial publications from the same research team using overlapped subjects. Our study was approved and reviewed by the institutional ethics committee of The First Affiliated Hospital of Nanjing Medical University and adhered to the tenets of the Declaration of Helsinki.

Data Collection and Quality Evaluation. Two investigators (X.C. and X.K.) independently reviewed all retrieved articles based on title, abstracts, and complete document if necessary. They also extracted data from each study separately with a customized datasheet. All data obtained from the two reviewers were compared. Disagreements were resolved through consensus with a senior reviewer (C.Z.). Data collected included: first author, year of publication, country of sample origin, ethnicity, genotyping method, demographics of subjects, average age and gender ratio of each studied group, allelic and genotypic distributions in cases and controls, adjusted OR and adjusted factors. The NOS (accessed via http://www.ncbi.nlm.nih. gov/books/NBK35156/) was applied for evaluation of the risk of biases for included studies.

Data Synthesis and Analysis. Hardy-Weinberg equilibrium was calculated using $\chi^{2}$ test to compare the genotypic frequencies of the $C 2$ rs547154 variant among the control subjects. Heterogeneity among studies was assessed using Cochran's Q 
statistic, and evaluated by the proportion of variation attributable to among-study heterogeneity, $I^{2}$. Heterogeneity was determined as indicated previously ${ }^{27}$.

The following five ORs and their 95\% CI were calculated using both random-effect (the DerSimonian and Laird method) ${ }^{28}$ and fixed-effect model (the Mantel-Haenszel method) ${ }^{29}$ : T versus $\mathrm{G}$ (allele model), TT versus GG (homozygous model), TG versus GG (heterozygous model), TT + TG versus GG (dominant model), and TT versus TG + GG (recessive model). Subgroup analysis was performed by ethnicity. Sensitivity was used to affirm the results by removing one individual study each time. Begg's funnel plot $^{30}$ and Egger's test ${ }^{31}$ were applied to appraise the publication bias and small-study effects. All analyses were conducted with Review Manager (version 5.2; Cochrane Collaboration, Oxford, UK; http://ims.cochrane.org/revman) and STATA software (version 12.0; StataCorp LP, College Station, TX). Alpha was set to 0.05 for two-sided test.

1. Koh, A. H. et al. Polypoidal choroidal vasculopathy: evidence-based guidelines for clinical diagnosis and treatment. Retina 33, 686-716 (2013).

2. Yannuzzi, L. A., Sorenson, J., Spaide, R. F. \& Lipson, B. Idiopathic polypoidal choroidal vasculopathy (IPCV). Retina 10, 1-8 (1990).

3. Nakashizuka, H. et al. Clinicopathologic findings in polypoidal choroidal vasculopathy. Invest Ophthalmol Vis Sci 49, 4729-4737 (2008).

4. Yannuzzi, L. A. et al. The expanding clinical spectrum of idiopathic polypoidal choroidal vasculopathy. Arch Ophthalmol 115, 478-485 (1997).

5. Lafaut, B. A., Leys, A. M., Snyers, B., Rasquin, F. \& De Laey, J. J. Polypoidal choroidal vasculopathy in Caucasians. Graefes Arch Clin Exp Ophthalmol 238, 752-759 (2000).

6. Ciardella, A. P., Donsoff, I. M., Huang, S. J., Costa, D. L. \& Yannuzzi, L. A. Polypoidal choroidal vasculopathy. Surv Ophthalmol 49, 25-37 (2004).

7. Chang, Y. C. \& Wu, W. C. Polypoidal choroidal vasculopathy in Taiwanese patients. Ophthalmic Surg Lasers Imaging 40, 576-581 (2009).

8. Terasaki, H., Miyake, Y., Suzuki, T., Nakamura, M. \& Nagasaka, T. Polypoidal choroidal vasculopathy treated with macular translocation: clinical pathological correlation. Br J Ophthalmol 86, 321-327 (2002).

9. Okubo, A., Sameshima, M., Uemura, A., Kanda, S. \& Ohba, N. Clinicopathological correlation of polypoidal choroidal vasculopathy revealed by ultrastructural study. Br J Ophthalmol 86, 1093-1098 (2002).

10. Wang, Z. Y. et al. Systematic review and meta-analysis of the association between complement factor $\mathrm{H} \mathrm{I} 62 \mathrm{~V}$ polymorphism and risk of polypoidal choroidal vasculopathy in Asian populations. PLoS One 9, e88324 (2014).

11. Yuan, D., Yuan, S. \& Liu, Q. The age-related maculopathy susceptibility 2 polymorphism and polypoidal choroidal vasculopathy in Asian populations: a meta-analysis. Ophthalmology 120, 2051-2057 (2013).

12. Sun, C., Zhao, M. \& Li, X. CFB/C2 gene polymorphisms and risk of age-related macular degeneration: a systematic review and meta-analysis. Curr Eye Res 37, 259-271 (2012).

13. Thakkinstian, A. et al. The association between complement component $2 /$ complement factor B polymorphisms and age-related macular degeneration: a HuGE review and meta-analysis. Am J Epidemiol 176, 361-372 (2012).

14. Lee, K. Y. et al. Association analysis of CFH, C2, BF, and HTRA1 gene polymorphisms in Chinese patients with polypoidal choroidal vasculopathy. Invest Ophthalmol Vis Sci 49, 2613-2619 (2008).

15. Kondo, N., Honda, S., Kuno, S. \& Negi, A. Role of RDBP and SKIV2L variants in the major histocompatibility complex class III region in polypoidal choroidal vasculopathy etiology. Ophthalmology 116, 1502-1509 (2009).

16. Lima, L. H. et al. Three major loci involved in age-related macular degeneration are also associated with polypoidal choroidal vasculopathy. Ophthalmology 117, 1567-1570 (2010)

17. Nakata, I. et al. Significance of C2/CFB variants in age-related macular degeneration and polypoidal choroidal vasculopathy in a Japanese population. Invest Ophthalmol Vis Sci 53, 794-798 (2012).

18. Tanaka, K. et al. Associations of complement factor B and complement component 2 genotypes with subtypes of polypoidal choroidal vasculopathy. BMC Ophthalmol 14, 83 (2014).

19. Bentley, D. R. \& Porter, R. R. Isolation of cDNA clones for human complement component C2. Proc Natl Acad Sci U S A 81, 1212-1215 (1984).
20. Bentley, D. R. Primary structure of human complement component C2. Homology to two unrelated protein families. Biochem J 239, 339-345 (1986).

21. Gehrs, K. M., Jackson, J. R., Brown, E. N., Allikmets, R. \& Hageman, G. S. Complement, age-related macular degeneration and a vision of the future. Arch Ophthalmol 128, 349-358 (2010).

22. Wetsel, R. A. et al. Type II human complement C2 deficiency. Allele-specific amino acid substitutions (Ser189 --> Phe; Gly444 --> Arg) cause impaired C2 secretion. J Biol Chem 271, 5824-5831 (1996).

23. Zhu, Z. B., Atkinson, T. P. \& Volanakis, J. E. A novel type II complement C2 deficiency allele in an African-American family. J Immunol 161, 578-584 (1998).

24. Chen, H. et al. Genetic associations in polypoidal choroidal vasculopathy: a systematic review and meta-analysis. Mol Vis 18, 816-829 (2012).

25. Liu, K. et al. Associations of the C2-CFB-RDBP-SKIV2L locus with age-related macular degeneration and polypoidal choroidal vasculopathy. Ophthalmology 120, 837-843 (2013).

26. Moher, D., Liberati, A., Tetzlaff, J. \& Altman, D. G. Preferred reporting items for systematic reviews and meta-analyses: the PRISMA statement. BMJ 339, b2535 (2009).

27. Chen, X. et al. Diabetes mellitus and risk of age-related macular degeneration: a systematic review and meta-analysis. PLoS One 9, e108196 (2014).

28. DerSimonian, R. \& Laird, N. Meta-analysis in clinical trials. Control Clin Trials 7, $177-188$ (1986)

29. Kuritz, S. J., Landis, J. R. \& Koch, G. G. A general overview of Mantel-Haenszel methods: applications and recent developments. Annu Rev Public Health $\mathbf{9}$, 123-160 (1988)

30. Begg, C. B. \& Mazumdar, M. Operating characteristics of a rank correlation test for publication bias. Biometrics 50, 1088-1101 (1994).

31. Egger, M., Davey Smith, G., Schneider, M. \& Minder, C. Bias in meta-analysis detected by a simple, graphical test. BMJ 315, 629-634 (1997).

\section{Acknowledgments}

This work was supported by National Key Basic Research Program of China (973 Program, 2013CB967500); National Natural Science Foundation of China (81222009 and 81170856); Thousand Youth Talents Program of China (to C. Z.); Jiangsu Outstanding Young Investigator Program (BK2012046); Jiangsu Province's Key Provincial Talents Program (RC201149); the Fundamental Research Funds of the State Key Laboratory of Ophthalmology (to C. Z.); Jiangsu Province's Scientific Research Innovation Program for Postgraduates (CXZZ13_0590); and A Project Funded by the Priority Academic Program Development of Jiangsu Higher Education Institutions (PAPD). The funders had no role in study design, data collection and analysis, decision to publish, or preparation of the manuscript.

\section{Author contributions}

Study design: X.C., K.Z. and C.Z. Collected the samples and performed the experiments: X.C. and X.K. Data interpretation and analysis: X.C. and X.K. Wrote the manuscript: X.C and C.Z. All authors have read and approved the final manuscript.

\section{Additional information}

Supplementary information accompanies this paper at http://www.nature.com/ scientificreports

Competing financial interests: The authors declare no competing financial interests.

How to cite this article: Chen, X., Kang, X., Zhao, K. \& Zhao, C. C2 rs547154 polymorphism and polypoidal choroidal vasculopathy susceptibility: a meta-analysis. Sci. Rep. 5, 8709 DOI:10.1038/srep08709 (2015)

This work is licensed under a Creative Commons Attribution 4.0 International License. The images or other third party material in this article are included in the article's Creative Commons license, unless indicated otherwise in the credit line; if the material is not included under the Creative Commons license, users will need to obtain permission from the license holder in order to reproduce the material. To view a copy of this license, visit http://creativecommons.org/licenses/by/4.0/ 